\title{
Exploring Barriers to 'Respondent Driven Sampling' in Sex Worker and Drug-Injecting Sex Worker Populations in Eastern Europe
}

\author{
Milena Simic, Lisa Grazina Johnston, Lucy Platt, Sladjana Baros, \\ Violeta Andjelkovic, Tom Novotny, and Tim Rhodes
}

\begin{abstract}
Respondent driven sampling (RDS) has been used in several counties to sample injecting drug users, sex workers ( $S W s$ ) and men who have sex with men and as a means of collecting behavioural and biological health data. We report on the use of RDS in three separate studies conducted among SWs between 2004 and 2005 in the Russian Federation, Serbia, and Montenegro. Findings suggest that there are limitations associated with the use of RDS in SW populations in these regions. Findings highlight three main factors that merit further investigation as a means of assessing the feasibility and appropriateness of RDS in this high risk population: the network characteristics of SWs; the appropriate level of participant incentives; and lack of service contact. The highly controlled and hidden nature of SW organizations and weak $S W$ social networks in the region can combine to undermine assumptions underpinning the feasibility of RDS approaches and potentially severely limit recruitment. We discuss the implications of these findings for recruitment and the use of monetary and non-monetary incentives in future RDS studies of $S W$ populations in Eastern Europe.
\end{abstract}

KEYWORDS Respondent driven sampling, Hidden populations, Russia, Serbia, Montenegro, Sex workers.

\section{INTRODUCTION}

In many countries in Eastern Europe large scale social and economic transition over the past 15 years has been associated with widespread poverty and increasing levels of unemployment and population migration, particularly among women. ${ }^{1,2}$ Such transition has also been associated with shifts in patterns of sexual behaviour and sexual relationships, including increased commercial sex work, the growth of informal sex work economies and associated human trafficking. ${ }^{3}$ Additionally, a number of countries in the former Soviet Union have experienced a rapid or explosive spread of HIV associated with injecting drug use. ${ }^{4-7}$ These epidemics are potentially exacerbated by close links between sex work and injecting drug

Simic, Platt, and Rhodes are with the The Centre for Research on Drugs and Health Behaviour, Department of Public Health and Policy, London School of Hygiene and Tropical Medicine, Keppel Street, London, WC1E 7HT, UK; Johnston (independent consultant) and Novotny are with the Institute for Global Health, University of California, San Francisco, CA, USA; Baros is with the Department of Anthropology, University of Belgrade, Belgrade, Serbia and Montenegro; Andjelkovic is with the International Aid Network, Belgrade, Serbia and Montenegro.

Correspondence: Tim Rhodes, PhD, The Centre for Research on Drugs and Health Behaviour, Department of Public Health and Policy, London School of Hygiene and Tropical Medicine, Keppel Street, London, WC1E 7HT, UK. (E-mail: tim.rhodes@lshtm.ac.uk) 
use. ${ }^{2,8-11}$ This has caused concerns that high levels of sexual mixing between injecting drug users (IDUs) and their sexual partners in settings of high HIV and sexual transmitted infection (STI) prevalence may transform HIV infection from concentrated epidemics among risk populations to generalised epidemics. ${ }^{9,10}$ It is therefore essential to assess the diffusion of HIV and related risk behaviour in hidden vulnerable populations, such as IDUs and sex workers (SWs), as accurately as possible.

There has been increased and recent interest in an approach to sampling hidden populations known as respondent driven sampling (RDS). ${ }^{12,13}$ One of the claimed advantages of RDS over other methods of sampling hidden populations, such as time-location sampling, is that it requires little in-depth formative research among study populations. ${ }^{13,14}$ RDS begins with a set number of non-randomly selected seeds (members of the target population). Seeds recruit their peers (other target population members who make up their social network) who in turn recruit their peers into the study. This occurs through successive waves of recruits which, it is argued, become increasingly more representative of the underlying population as the recruitment progresses. Since RDS claims to generate a representative sample from non-randomly selected seeds, many researchers have assumed that sampling can proceed without detailed prior knowledge about the local target population. $^{13,14}$

RDS has proved feasible and successful in recruiting hidden populations of IDUs in a variety of settings, resulting in the rapid acquisition of long and varied recruitment chains. ${ }^{11,14-19}$ RDS has also been used in several countries to collect both behavioural and biological data from SWs (e.g., Vietnam, India) and men who have sex with men (e.g., Bangladesh, Cambodia, Uganda, United States). ${ }^{20-24}$ However, little is known about the feasibility of RDS as a method of recruiting hidden populations of SWs in settings where the organisation of sex work and access to SWs is highly controlled and where there is little or no contact between the target population and local services. Drawing upon three case studies in Eastern Europe targeting SWs and IDUs involved in SW (IDU/SWs), we explore the feasibility of RDS, identifying some barriers to successful recruitment. These findings have implications for the direction of future research exploring the feasibility of RDS methods.

\section{METHODS}

Using RDS, data on HIV prevalence and associated risk factors were gathered from SWs in Serbia and Montenegro and from IDUs involved in sex work in Russia (see Table 1). SWs in Serbia and Montenegro were given three uniquely numbered coupons with which to recruit other SWs. In Russia, although no RDS study directly targeting SWs was carried out, RDS was used to sample SWs/IDUs in an RDS study among IDUs. Incentives were provided to participants completing the survey (primary incentive) and for recruiting other eligible participants (secondary incentive). Participants in Belgrade were also offered a rapid HIV test and pre- and post-test voluntary and confidential counselling (VCT). Those with preliminarypositive tests for HIV were referred to a local clinic for confirmatory testing. The studies were undertaken by local fieldwork teams coordinated as part of larger internationally funded research initiatives.

All studies were approved by appropriate local ethical committees and international human subject review boards. Participants were required to agree to 


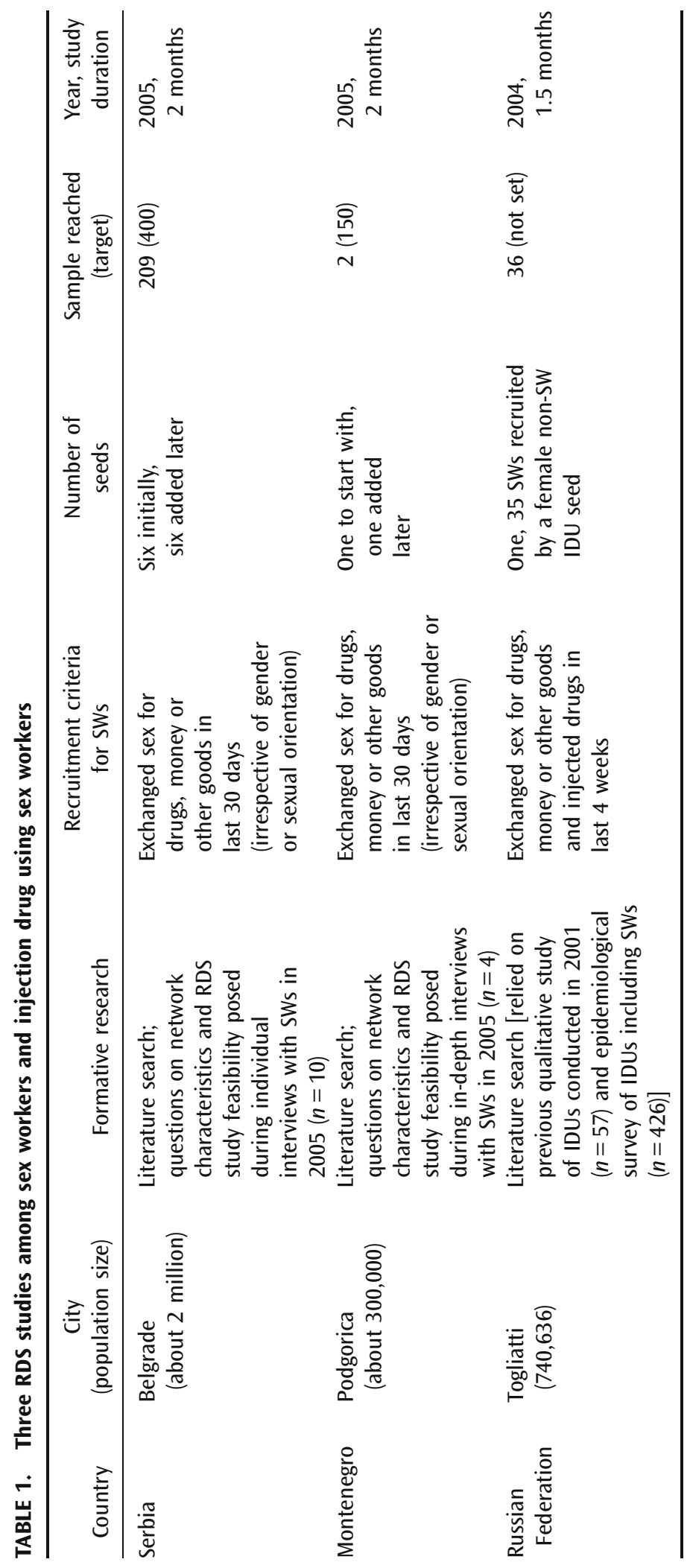


an informed consent before participating. Participants were not linked to the study with any personal identifiers, and all participant information was kept confidential and anonymous.

Prior to data collection, formative research, comprising a combination of key informant interviews and a literature review, was undertaken to assess the local feasibility of the RDS study identifying the optimal study set up (Table 1, column 3). The formative research elicited data on size estimates of the sex work population; description of how sex work is organised (sectors of sex work, freedom of movement, average earnings and prices charged for services in each sector); and the legal situation surrounding sex work. In Serbia and Montenegro, researchers also asked SWs about the size of their social networks in order to fine-tune questions on which RDS analysis would be based. A social network was defined as the number of SWs, 18 years and older, each SW knows and has seen in the past week, month, three months, six months, or year.

\section{RESULTS}

As shown in Table 1, a total of 209 SWs were recruited in Belgrade (Serbia), two in Podgorica (Montenegro) and 36 in Togliatti (Russian Federation).

\section{Case 1: Serbia}

RDS was conducted in the autumn of 2005 in Belgrade, Serbia's capital, by a local fieldwork team with a limited access to SW networks. Estimates of the size of the SW population in the municipality, based on police records and anecdotal estimates, vary widely from 900 to 3,000 SWs. ${ }^{2,24,25}$

Sex work is illegal. Findings from in-depth interviews of SWs suggest that there are approximately ten areas located near the main transit routes in and out of Belgrade where street SWs operate in small groups. Qualitative data suggests that the majority of SWs operate independently, advertising their services in newspapers or through referrals via existing client networks, potentially making them harder to reach through RDS.

Formative interviews also found that there was little mixing of SWs across different sex work sectors (street, agency, and SWs working independently), among street SWs themselves (transvestite, Roma and Serbian/Montenegrin heterosexual SWs) and among SWs working independently. However, in addition to a lack of strong connection among SWs and their independent working situations, the study team undertaking formative qualitative research associated SWs' reluctance to participate in the study to perceptions of inadequate incentive (10 euros for 1 hour of their time) and a general mistrust associated with coming into contact with 'official' agencies. However, the same interviews suggested that in principle SWs would be interested in participating in a survey involving the collection of both behavioural and biological data through HIV testing. Interviewees indicated that they knew approximately 20 other SWs, which again suggested that RDS would be feasible.

The RDS study had a target of 400 SWs, both male and female. The sample needed to be large enough for a statistically reliable analysis of risk factors and HIV prevalence, should RDS analysis show that the sample is reasonably representative. The interview site was on the premises of a non-governmental organisation (NGO) located in central Belgrade easily accessible by public transport. It was a part of a large apartment block with a separate entrance. The site was open everyday from 3 to 9 p.m. and could accommodate three to four participants an hour because of the 
computer administered questionnaire. After two months, the study ceased, having reached only 209 SWs. In total, 12 seeds were used during the study. Of the initial six seeds only three produced any subsequent waves. The rate of recruiting increased noticeably after an RDS study of IDUs being implemented at the same site had ceased. Due to the difficulties experienced by the research team in locating new seeds through other means, four participants with dense social networks were drawn from the seventh wave of two of the most productive recruitment chains and used as new seeds. This resulted in two more productive chains that ultimately reached seven waves.

The average SW network size reported during the study was 12 . In response to the question, "where do you most often solicit your clients?," the majority of SWs replied that they solicited on the street $(43 \%)$, followed by referral from an agency, pimps, friends or other SWs $(28.9 \%)$ and by telephone $(25.2 \%)$. Importantly, the take-up of VCT provided as part of the RDS study was high $(71.8 \%)$, and SWs indicated that they participated because of the HIV testing, rather than the monetary incentive.

\section{Case 2: Montenegro}

There are no estimates of the number of SWs in Podgorica, the Republic's capital. Sex work is ostensibly illegal. Through formative rapid assessment no street-based SWs were identified and the majority of SWs were found to work independently from their homes. Key informant interviews (IDUs, ex-IDUs, taxi drivers, public health officials, NGO representatives and journalists) identified the only semipublic sex work sites as being approximately six illegal brothels, registered as lap dancing venues, in which most of the SWs also lived. At least one such brothel was found not to allow SWs to leave the premises. Indications were that local police also 'moonlighted' as security guards in these brothels. Other links between brothels and public services included taxi drivers, beauty salons and hotels. The intersection of the police and sex work networks, the small size of the city and the high number of implicated actors suggested that SWs could be easily monitored and controlled.

Previous attempts by the local NGOs to conduct research among SWs in Podgorica had failed. Key informants, interviewed during formative rapid assessment, reported knowing at least one SW or their pimps but in almost all cases introductions were denied or never materialized. Those conducting the formative rapid assessment received strong advice from local experts and key informants-including NGOs, police, and taxi drivers-against approaching brothel owners because of the highly controlled nature of SW venues in the city. After two weeks of trying to make a contact, four SWs were accessed via their drug user networks, the only entry points into SW networks available to researchers. These SWs were interviewed at their place of work (brothel and pimp's flat). Each of the four interviewed SWs independently estimated that there were approximately 200 SWs in the city, many of them also drug injectors, most of whom worked independently, advertising their services via newspapers and television and via client recommendation but who were not open about their SW status. All four SWs interviewed indicated that they would participate in a RDS study and would invite others to do so, and each estimated they had personal regular contact with between five and ten SWs.

The four SWs participating in the formative rapid assessment were invited to participate in the RDS study as seeds. Only one of the four participated but failed to 
recruit anyone else. She reported having had contact with ten SWs in 4 weeks, but no additional seeds were secured. Despite recruiting an additional SW (who required complete anonymity fearing being identified as a SW by others to his family and who had not seen other SWs in the past month), the RDS study failed to identify a sufficient pool of seeds willing to participate.

\section{Case 3: Russia}

The RDS study of IDUs and IDU/SWs was conducted in May 2004 in Togliatti City. Togliatti City is the second city of Samara Region situated approximately 1,000 km south of Moscow. Togliatti was selected as a study site because evidence suggests a recent increase in the number of IDUs and SWs and an explosive spread of HIV associated with injecting drug use. . $^{6} 11$

Approximately 2,000 SWs are estimated to operate in Togliatti, half of whom work from the street. ${ }^{6}$ Despite ambiguous Russian legislation towards sex work, it is not tolerated by the wider community or the police. The city has been noted for its intense street policing of drug users and SWs, often involving fines levied under administrative codes, such as possession of drugs, causing a public nuisance, or lack of official residency permit, rather than criminal codes. ${ }^{26,27}$ Street-based SWs in Togliatti operate openly along the main roads and highways. Two popular locations are Moskovsky Prospect where approximately 70 women are working at any one time, and Pobeda Street where about 100 women work.

In a study conducted in Togliatti City in $2001,50 \%$ of female IDUs $(n=155)$ reported ever exchanging sex for goods or money, of whom $86 \%(n=66)$ were currently SWs. ${ }^{6}$ Although no stand-alone RDS studies of SWs have been conducted in Russia, attempts have been made to boost participation of IDU/SWs in RDS studies of IDUs motivated by the evidence of high proportion of SWs who also inject drugs in other cities. ${ }^{28-30}$

A cross-sectional survey of IDUs and female IDU/SWs was undertaken in Togliatti in 2004 as a follow up to the 2001 baseline survey. A total of 476 IDUs were sampled during a 5-week period in May 2004 using RDS. Among these, 55 $(11 \%)$ reported ever exchanging sex for money, drugs or goods, and of these, 36 in the past month, which was lower than expected. At the start of the field work, seven seeds were selected, of which one seed was female who had exchanged sex in the past month. SWs were explicitly asked to recruit other SWs, and non-SWs were encouraged to recruit either SWs or non-SWs (Table 1, column 4). This first SW seed produced no recruits and was lost to follow up. Another female non-SW started a chain that recruited a total of 22 female IDUs who had ever exchanged sex, of whom 13 had exchanged sex in the past month. This female non-SW seed produced a total of 135 recruits by the end of the study equivalent to $28 \%$ of the total sample, of which $15 \%(20)$ were SWs and who reported working on the street. Among the other seeds, only a handful of current SWs were recruited $(n=4)$. Towards the end of the project more coupons were distributed to female SWs in an attempt to increase the SW sample $(n=5)$, none of which produced any recruits. In order to increase the sample of SWs in the survey, 21 SWs were subsequently identified and interviewed without coupons.

Data on the number of female SWs within an individual's IDU network were unfortunately not provided. The mean IDU network size among SWs was 19.5 (SD 14.2) and among non-SWs 27.1 (SD 33.0). Only one SW recruited through RDS reported working from an apartment; all others were street-based SWs. We expected that RDS would enable us to recruit more hidden populations of SWs 
such as those working from apartments or hotels. The fact that SW seeds failed to produce any waves of SWs or were lost to follow up, indicates that SW networks may not be as well connected.

Field notes show that initial attempts to access street SWs at Moskovsky Prospect and Pobeda Street had failed primary because of intensified police action in the area. A local officer of the Federal Security Service believed he had been recently infected with HIV by a SW from the area, and, in revenge, he and his colleagues enforced a repressive policy of fines and arrests among SWs. Recruiting SWs towards the end of the survey was more successful when policing practices returned to normal. Meanwhile, the sample target was reached for non-SW IDUs.

\section{DISCUSSION}

Despite undertaking formative research in the planning of these RDS studies, we found that RDS methods were unable to attain sufficient samples of SWs in Serbia, Montenegro, and Russia. A number of inter-related factors emerged as important to the success of RDS: low motivation of SWs to participate due to their perception of inadequate incentive; low-density SW networks combined with high level of independence in the organisation of sex work; a tightly controlled small number of sex work venues; lack of contact between SWs and local services in the context of high levels of mistrust of 'official' agencies; and difficulties emerging in the organisation of the RDS studies of SWs at the same time as a parallel RDS study of IDUs.

Whereas in other settings (e.g., Vietnam, north-eastern India, Brazil) SWs have reacted favourably to nominal monetary incentives in RDS studies, including incentives which fall below the rate charged for sex, ${ }^{16,17}$ it is possible that the incentives offered were perceived as being too low. In order to earn primary and secondary incentives, which together in all three studies amounted to about a halfan-hour SW's rate, they had to leave their work and travel to and from the fixed interview site at least twice plus spend at least an hour involved in the study. It is possible that SWs in Eastern Europe may be less interested in the incentives offered since they have comparatively higher earnings.

Indications are that free HIV/STI health assessments and referral for treatment should be explored in addition to the incentive. The high acceptance of VCT in Belgrade, for example, is a good example of how provision of services can be more effective than monetary incentives in recruiting survey participants. If incentives are purely monetary, they should be adequate to entice SWs to participate; yet this is often not feasible given the risk of high incentives also encouraging the attempted participation of non-SWs.

Unlike other RDS studies conducted in Vietnam, ${ }^{16}$ SWs in Belgrade felt uncomfortable being interviewed at a location where a concurrent study of IDUs was being conducted. Based on anecdotal information and the fact that SWs in Belgrade started participating in the study once the IDU study had finished, we believe that SWs were dissuaded from going to a location that was used by IDUs. This problem can be assessed during formative research using focus groups with SWs to assess whether they would feel comfortable sitting in a waiting room with a group of male and female IDUs.

Perhaps the main reason for the poor success of RDS recruitment among SWs in these three settings is the characteristics of SW networks. RDS methodology 
requires that the target population form sufficiently large and dense networks. However, the information on the network sizes of a few SWs gathered through our formative research are not easy to interpret since there is no known cut-off point for determining an appropriate network size or density for RDS. Based on the results of our studies, SWs in our three study areas often work independently, thus forming smaller, more isolated, networks. Those working on the street and in brothels are also under tight control by managers, pimps and police thus restricting their movement and weakening network density. On the other hand, prevalent societal norms-a form of social control-prevent SWs who work independently from revealing their SW status and participating. The low recruitment rate encountered in our studies might be overcome by allowing studies to run for a much longer time-perhaps as long as six months or a year-allowing SWs to gain trust in the investigators.

In the studies described above, interview sites were rented and staff employed for a fixed amount of time. A preferable setting is one with on-going services for SWs that can be used as an interview site for a long period of time allowing recruits to come at a slow rate. However, time was unlikely a major factor contributing to difficulties in RDS recruitment in the cities where our studies were conducted.

The dearth of well-established projects targeting SWs in Eastern Europe means that greater effort should be invested prior to RDS studies to identify a greater number of seeds and engender SWs' trust. In some cases existence of a wellestablished service with established contacts with local SW communities might be a decisive factor. For example, this was the case in a RDS study among SWs in Tbilisy, Georgia (January 2006, written communication, Johnston LG). In addition to promoting trust, such service organisations can provide additional networking opportunities for loosely connected SWs.

\section{CONCLUSION}

Given the cost of conducting second-generation sero-surveillance among highly vulnerable populations, formative research is important for establishing the appropriate type of sampling and reward system. We found the making available free and confidential HIV testing services was an effective incentive, and perhaps more so than monetary incentives alone. Although formative research would require some additional costs, it can increase efficiency in a more extensive and successful study.

During formative research, we suggest that policy makers, service providers and target populations be consulted to determine the visibility of sex work, as low visibility can be associated with tight control of the sex work industry and weak networking among SWs, indicating difficulties using RDS. If researchers find it difficult to recruit SWs for formative study, the RDS study is likely to fail. Depending on the timescale, funding and interest among existing service providers, additional services may be provided well ahead of a RDS study in order to access SW respondents.

Further investigation is needed into the ethnography of SW networks in this region, especially on network composition and service access. In the case of IDUs who exchange sex for money, goods, or drugs, it is important to understand their primary network linkage-injecting drug use or sex work-and how they represent their "other identity" within each network. Incentives need to be better identified in advance of RDS studies, specifically as they relate to level of income, opportunity 
costs, and trust in the investigators and seeds. Without this cultural and legal sensitivity, surveillance for HIV among this vulnerable population will not provide adequate information on epidemic spread or program effectiveness.

\section{ACKNOWLEDGEMENTS}

These studies were funded by the UK Department of International Development and, in Serbia and Montenegro, in collaboration with the U.S. Centers for Disease Control and Prevention-Global AIDS Program through the University of California, San Francisco. The authors wish to thank the Institutes for Public Health in Serbia and Montenegro, and non-governmental organizations, International Aid Network in Belgrade and Juventas in Podgorica, for their contributions to this study. We also thank the sex workers who participated in these studies and to the peer outreach workers who helped select our participants.

\section{REFERENCES}

1. Brainerd WE. Losers in Russia's economic transition. Am Econ Rev. 1998;88:1094-1116.

2. Rhodes T, Simic M. Transition and the HIV risk environment. Br Med J. 2005;331:220-223.

3. Cucic V, Bjegovic V, Ignjatovic-Ristic D, Ilic B, Beara V. Rapid assessment and response (RAR) on HIV/AIDS among especially vulnerable young people in Serbia. Belgrade, Serbia: UNICEF and University Belgrade, Institute for Social Medicine; 2002.

4. Hamers F, Infuso A, Alix J, Downs AM. Current situation and regional perspective on HIV/AIDS surveillance in Europe. J Acquir Immune Defic Syndr. 2003;32(Supplement 1):S39-S48.

5. Rhodes T, Ball B, Stimson V, et al. HIV infection associated with drug injecting in the newly independent states, eastern Europe: the social and economic context of epidemics. Addiction. 1999;94(9):1323-1336.

6. Rhodes T, Lowndes CM, Judd A, et al. Explosive spread and high prevalence of HIV infection among injecting drug users in Togliatti City, Russia. AIDS. 2002;16:F25-F31.

7. Rhodes T, Sarang A, Bobrik A, Bobkov E, Platt L. HIV transmission and HIV prevention associated with injecting drug use in the Russian Federation. Int J Drug Policy. 2004; 15:1-16.

8. Aral SO, St. Lawrence JS, Dyatlov R, Kozlov A. Commercial sex work, drug use, and sexually transmitted infections in St. Petersburg, Russia. Soc Sci Med. 2005;60:21812190.

9. Pokrovsky V, Ladnaya N, Sokolova E. HIV epidemic in Russia and Eastern Europe: are there any predictors for further heterosexual spreading? Paper presented at 14th International AIDS Conference, July 2002, Spain, Barcelona.

10. Grassly N, Lowndes CM, Rhodes T, et al. Modelling emerging HIV epidemics: the role of injecting drug use and sexual transmission in the Russian Federation, China and India. Int J Drug Policy. 2003;13:25-43.

11. Platt L, Rhodes T, Judd A, et al. Syphilis among injecting drug users in three cities in Russia: the effect of sex work. Am J Public Health. In press

12. Magnani R, Sabin K, Saidel T, Heckathorn D. Sampling hard to reach and hidden populations for HIV surveillance. AIDS. 2005;19(Supplement 2):S67-S72.

13. Semaan S, Lauby J, Liebman J. Street and network sampling in evaluation studies of HIV risk-reduction interventions. AIDS Rev. 2002;4:213-223.

14. Heckathorn DD. Respondent driven sampling: a new approach to the study of hidden populations. Soc Probl. 1997;44(2):174-199.

15. Abdul-Quader SL, Heckathorn DD, Mcknight C, et al. Effectiveness of respondent driven sampling for recruiting drug users in New York City: findings from a pilot study. $J$ Urban Health. 2006;83:459-476. 
16. Johnston LG, Huong PT, Hien MT, et al. HIV prevalence and risk factors among injecting drug users in Ho Chi Minh City, Vietnam. Paper presented at the International Conference for the reduction of harm among injecting drug users. Belfast, Ireland; March 2005.

17. Salganik MJ, Heckathorn DD. Sampling and estimation in hidden populations using respondent-driven sampling. Sociol Method. 2004;34:193-239.

18. Heckathorn DD. Respondent driven sampling II: deriving valid population estimates from chain-referral samples of hidden populations. Soc Probl. 2002a;49(1):11-34.

19. Heckathorn D, Semaan S, Broadhead RS, Hughes JJ. Extensions of respondent-driven sampling: a new approach to the study of injection drug users aged 18-25. AIDS Behav. $2002 b ; 6(1): 55-67$.

20. Johnston LG. Behavioral surveillance using respondent driven sampling among commercial sex workers in Ho Chi Minh City, Vietnam. Ph.D. dissertation. Tulane University School of Public Health and Tropical Medicine, Louisiana; 2005.

21. Johnston LG. Update on Conducting Respondent Driven Sampling in Developing Countries. Notes from a Regional Training Among Representatives from Centers for Disease Control and Prevention, Family Health International and University of California, San Francisco. Bangkok, Thailand; 2005, August 8-12.

22. Ramirez-Valles J, Heckathorn DD, Vazquez R, Diaz RM, Campbell RT. From networks to populations: the development and application of respondent-driven sampling among IDUs and Latino gay men. AIDS Behav. 2005;9(4):387-402.

23. Centers for Disease Control and Prevention. Multi-Agency Technical Consultation on Sampling Most at Risk Populations. Summary report Atlanta, GA; 2004.

24. Radio Television Serbia. May 12, 2005.

25. Nedeljni telegraf. February 20, 2002.

26. Rhodes T, Mikhailova L, Sarang A, et al. Situational factors associated with drug injecting, risk reduction and syringe exchange practices in Togliatti City, Russian Federation: a qualitative study of micro risk environment. Soc Sci Med. 2003;57:39-54.

27. Rhodes T, Platt L, Maximova S, et al. Prevalence of HCV, HIV and syphilis among injecting drug users in Russia: multi-city study. Addiction. 2006;101:252-266.

28. Benotsch EG, Somlai AM, Pinkerton SD, et al. Drug use and sexual risk behaviours among female Russian IDUs who exchange sex for money or drugs. Int J STD AIDS. 2004;15:343-347.

29. Abdala N, Carney JN, Durante AJ, et al. Estimating the prevalence of syringe-borne and sexually transmitted diseases among injection drug users in St Petersburg, Russia. Int J STD AIDS. 2003;14:697-703.

30. Karapetyan AF, Sokolovsky YV, Araviyskaya ER, et al. Syphilis among intravenous drugusing population: epidemiological situation in St Petersburg, Russia. Int J STD AIDS. 2002;13:618-623. 